\title{
Lower Bounds on Revenue of Approximately Optimal Auctions
}

\author{
Balasubramanian Sivan $^{1 \star}$, Vasilis Syrgkanis ${ }^{2 \star \star}$, and Omer Tamuz ${ }^{3 \star \star \star}$ \\ 1 Computer Sciences Dept., University of Winsconsin-Madison \\ balu2901@cs.wisc.edu \\ 2 Dept. of Computer Science, Cornell University, Ithaca, NY, USA \\ vasilis@cs. cornell.edu \\ 3 Weizmann Institute, Rehovot 76100, Israel \\ omert.tamuz@weizmann.ac.il
}

\begin{abstract}
We obtain revenue guarantees for the simple pricing mechanism of a single posted price, in terms of a natural parameter of the distribution of buyers' valuations. Our revenue guarantee applies to the single item $n$ buyers setting, with values drawn from an arbitrary joint distribution. Specifically, we show that a single price drawn from the distribution of the maximum valuation $V_{\max }=\max \left\{V_{1}, V_{2}, \ldots, V_{n}\right\}$ achieves a revenue of at least a $\frac{1}{e}$ fraction of the geometric expecation of $V_{\max }$. This generic bound is a measure of how revenue improves/degrades as a function of the concentration/spread of $V_{\max }$.

We further show that in absence of buyers' valuation distributions, recruiting an additional set of identical bidders will yield a similar guarantee on revenue. Finally, our bound also gives a measure of the extent to which one can simultaneously approximate welfare and revenue in terms of the concentration/spread of $V_{\max }$.
\end{abstract}

Keywords: Revenue, Auction, Geometric expectation, Single posted price

\section{Introduction}

Here is a natural pricing problem: A single item is to be sold to one among $n$ buyers. Buyers' valuations are drawn from some known joint distribution. How good a revenue can be achieved by posting a single price for all the buyers, and giving the item to the first buyer whose value exceeds the price? Can we lower bound the revenue in terms of some properties of the distribution? Such a single pricing scheme is often the only option available, for several natural reasons. In

* This work was done while the author was an intern at Microsoft Research.

** Part of this work was done while the author was an intern at Microsoft Research. Supported in part by ONR grant N00014-98-1-0589 and a Simons Graduate Fellowship.

$\star \star \star$ Part of this work was done while the author was a visitor at Microsoft Research. Supported in part by a Google Europe fellowship in Social Computing. 
many situations, it is illegal or not in good taste to price discriminate between buyers; furthermore often it is not possible to implement a pricing scheme with multiple prices.

We define the geometric expectation of a random variable before describing our result: the geometric expectation of a random variable $X$ is given by $e^{\mathbb{E}[\log (X)]}$ (see, e.g., [5]). The geometric expectation is always lower than the expectation, and the more concentrated the distribution, the closer they are; indeed, the ratio between the geometric expectation and the expectation is a natural measure of concentration around the mean. We illustrate how the ratio of geometric and actual expectations captures the spread of a random variable through an example in Section 2.

Constant fraction of geometric expectation. We show that a single price obtains a $\frac{1}{e}$ fraction of the geometric expectation of the maximum among the $n$ valuations $\left(V_{1}, \ldots, V_{n}\right)$, i.e. geometric expectation of $V_{\max }=\max \left\{V_{1}, \ldots, V_{n}\right\}$. Thus for distributions that are concentrated enough to have a geometric expectation of $V_{\max }$ that is close to the expectation of $V_{\max }$, a single pricing scheme extracts a good fraction of the social surplus. In particular, when the ratio of geometric and actual expectations is larger than $e / 4$, our revenue guarantee is larger than a $1 / 4$ fraction of the welfare (and hence the optimal revenue), thus beating the currently best known bound of $1 / 4$ by Hartline and Roughgarden [4]. In the special case when the distribution of $V_{\max }$ satisfies the monotone hazard rate (MHR) property, a single price can extract a $\frac{1}{e}$ fraction of the expected value of $V_{\max }([3])$. However, since several natural distributions fail to satisfy the MHR property, establishing a generic revenue guarantee in terms of the geometric expectation, and then bounding the ratio of the geometric and actual expectation is a useful route. For instance, in Section 2 we compute this ratio for power law distributions (which do not satisfy the MHR property) and show that for all exponents $m \geq 1.56$ this ratio is larger than $e / 4$ thus beating the currently known bound.

Why geometric expectation?

1. Since the concentration of a distribution is a crucial property in determining what fraction of welfare (expectation of $V_{\max }$ ) can be extracted as revenue, it is natural to develop revenue guarantees expressed in terms of some measure of concentration.

2. While there are several useful measures of concentration for different contexts, in this work we suggest that for revenue in auctions the ratio of the geometric and actual expectations is both a generic and a useful measure as explained in the previous paragraph, for some distributions our revenue guarantees are the best known so far.

3. The ratio of the two expectations is a dimensionless quantity (i.e., scale free).

Second price auction with an anonymous reserve price. A natural corollary of the lower bound on single pricing scheme's revenue is that the second price auction (or the Vickrey auction) with a single anonymous reserve obtains a fraction $\frac{1}{e}$ of 
the geometric expectation of $V_{\max }$. When buyers' distributions are independent and satisfy a technical regularity condition, Hartline and Roughgarden [4] show that the second price auction with a single anonymous reserve price obtains a four approximation to the optimal revenue obtainable. Here again, our result shows that for more general settings, where bidders values could be arbitrarily correlated, Vickrey auction with a single anonymous reserve price guarantees a $\frac{1}{e}$ fraction of geometric expectation of $V_{\max }$.

Second price auction with additional bidders. When estimating the distribution is not feasible (and hence computing the reserve price is not feasible), a natural substitute is to recruit extra bidders to participate in the auction to increase competition. We show that if we recruit another set of bidders distributed identically to the first set of $n$ bidders, and run the second price auction on the $2 n$ bidders, the expected revenue is at least a $\frac{2}{e}$ fraction of the geometric expectation of $V_{\max }$. As in the previous result, for the special case of independent distributions that satisfy the regularity condition, Hartline and Roughgarden [4] show that recruiting another set of $n$ bidders identical to the given $n$ bidders obtains at least half of the optimal revenue; our result gives a generic lower bound for arbitrary joint distributions.

In the course of proving this result we also prove the following result: in the single pricing scheme result, the optimal single price to choose is clearly the monopoly price of the distribution of $V_{\max }$. However we show that a random

price drawn from the distribution of $V_{\max }$ also achieves a $\frac{1}{e}$ fraction of geometric expectation of $V_{\max }$.

Related Work. For the special single buyer case, Tamuz [6] showed that the monopoly price obtains a constant fraction of the geometric expectation of the buyer's value. We primarily extend this result by showing that for the $n$ buyer setting, apart from the monopoly reserve price of $V_{\max }$, a random price drawn from the distribution of $V_{\max }$ also gives a $\frac{1}{e}$ fraction of geometric expectation of $V_{\max }$. This is important for showing our result by recruiting extra bidders. Daskalakis and Pierrakos [2] study simultaneous approximations to welfare and revenue for settings with independent distributions that satisfy the technical regularity condition. They show that Vickrey auction with non-anonymous reserve prices achieves a $\frac{1}{5}$ of the optimal revenue and welfare in such settings. Here again, for more general settings with arbitrarily correlated values, our result gives a measure how the quality of such simultaneous approximations degrades with the spread of $V_{\max }$. The work of Hartline and Roughgarden [4] on second price auction with anonymous reserve price / extra bidders has been discussed already.

\section{Definitions and Main Theorem}

Consider the standard auction-theoretic problem of selling a single item among $n$ buyers. Each buyer $i$ has a private (non-negative) valuation $V_{i}$ for receiving 
the item. Buyers are risk neutral with utility $u_{i}=V_{i} x_{i}-p_{i}$, where $x_{i}$ is the probability of buyer $i$ getting the item and $p_{i}$ is the price he pays. The valuation profile $\left(V_{1}, V_{2}, \ldots, V_{n}\right)$ of the buyers is drawn from some arbitrary joint distribution that is known to the auctioneer. Let $V_{\max }=\max _{i} V_{i}$ be the random variable that denotes the maximum value among the $n$ bidders. We denote with $F_{\max }$ the cumulative density function of the distribution of $V_{\max }$.

Definition 1. For a positive random variable $X$, the geometric expectation $\mathbb{G}[X]$ is defined as:

$$
\mathbb{G}[X]=\exp (\mathbb{E}[\log X])
$$

We note that by Jensen's inequality $\mathbb{G}[X] \leq \mathbb{E}[X]$ and that equality is achieved only when $X$ is a deterministic random variable. Further, as noted in the introduction, the ratio of geometric and actual expectations of a random variable is a useful measure of concentration around the mean. We illustrate this point through an example.

Example 2. Consider the family $F_{m}(x)=1-1 / x^{m}$ of power-law distributions for $m \geq 1$. As $m$ increases the tail of the distribution decays faster, and thus we expect the geometric expectation to be closer to the actual expectation. Indeed, the geometric expectation of such a random variable can be computed to be $e^{1 / m}$ and the actual expectation to be $\frac{m}{m-1}$. The ratio $e^{1 / m}(1-1 / m)$ is an increasing function of $m$. It reaches 1 at $m=\infty$, i.e., when the distribution becomes a point-mass fully concentrated at 1 . The special case of $m=1$ gives the equal-revenue distribution, where the geometric expectation equals $e$ and the actual expectation is infinity. However this infinite gap (or the zero ratio) quickly vanishes as $m$ grows; at $m=1.56$, the ratio already crosses $e / 4$ thus making our revenue guarantee better than the current best $1 / 4$ of optimal revenue; at $m=4$, the ratio already equals 0.963 .

For a random variable $X$ drawn from distribution $F$, define $\mathbb{R}_{p}[X]$ as:

$$
\mathbb{R}_{p}[X]=p \mathbb{P}[X \geq p] \geq p \mathbb{P}[X>p]=p(1-F(p))
$$

If $X$ is the valuation of a buyer, $\mathbb{R}_{p}[X]$ is the expected revenue obtained by posting a price of $p$ for this buyer. Therefore $\mathbb{R}_{p}\left[V_{\max }\right]$ is the revenue of a pricing scheme that posts a single price $p$ for $n$ buyers with values $V_{1}, \ldots, V_{n}$ and $V_{\max }=$ $\max \left\{V_{1}, \ldots, V_{n}\right\}$.

We show that the revenue of a posted price mechanism with a single price drawn randomly from the distribution of $V_{\max }$, achieves a revenue that is at least a $\frac{1}{e}$ fraction of the geometric expectation of $V_{\max }$, or equivalently a $\frac{1}{e}$ fraction of the geometric expectation of the social surplus.

Theorem 3 (Main Theorem). Let $r$ be a random price drawn from the distribution of $V_{\max }$. Then:

$$
\mathbb{E}_{r}\left[\mathbb{R}_{r}\left[V_{\max }\right]\right] \geq \frac{1}{e} \mathbb{G}\left[V_{\max }\right] .
$$


Proof. By the definition of $\mathbb{R}_{r}[V]$ we have:

$$
\mathbb{E}_{r}\left[\mathbb{R}_{r}\left[V_{\text {max }}\right]\right] \geq \mathbb{E}_{r}\left[r\left(1-F_{\max }(r)\right)\right] .
$$

By taking logs on both the of the above equation, and using Jensen's inequality we get:

$$
\begin{aligned}
\log \left(\mathbb{E}_{r}\left[\mathbb{R}_{r}\left[V_{\max }\right]\right]\right) & \geq \log \left(\mathbb{E}_{r}\left[r\left(1-F_{\max }(r)\right)\right]\right) \\
& \geq \mathbb{E}_{r}\left[\log \left(r\left(1-F_{\max }(r)\right)\right)\right] \\
& =\mathbb{E}_{r}[\log (r)]+\mathbb{E}_{r}\left[\log \left(1-F_{\max }(r)\right)\right] .
\end{aligned}
$$

For any positive random variable $X$ drawn from a distribution $F$ we have:

$$
\mathbb{E}[\log (1-F(X))]=\int_{-\infty}^{\infty} \log (1-F(x)) d F(x)=\int_{0}^{1} \log (1-y) d y=-1 .
$$

So we have:

$$
\begin{aligned}
\log \left(\mathbb{E}_{r}\left[\mathbb{R}_{r}\left[V_{\max }\right]\right]\right) & \geq \mathbb{E}_{r}[\log (r)]-1 \\
\mathbb{E}_{r}\left[\mathbb{R}_{r}\left[V_{\text {max }}\right]\right] & \geq \frac{1}{e} \exp \left(\mathbb{E}_{r}[\log (r)]=\frac{1}{e} \mathbb{G}\left[V_{\max }\right] .\right.
\end{aligned}
$$

where the last equality follows from the fact that the random reserve $r$ is drawn from $F_{\max }$.

Since a random price drawn from $F_{\max }$ achieves this revenue, it follows that there exists a deterministic price that achieves this revenue and hence the best deterministic price will achieve the same.

We define the monopoly price $\eta_{F}$ of a distribution $F$ to be the optimal posted price in a single buyer setting when the buyer's valuation is drawn from distribution $F$, i.e.:

$$
\eta_{F}=\arg \sup _{r} r(1-F(r))
$$

So a direct corollary of our main theorem is the following:

Corollary 4. Let $\eta_{\max }$ be the monopoly price of distribution $F_{\max }$. Then:

$$
\mathbb{R}_{\eta_{\max }}\left[V_{\max }\right] \geq \frac{1}{e} \mathbb{G}\left[V_{\max }\right]
$$

\section{Applications to Approximations in Mechanisms Design}

Single Reserve Mechanisms for Non-iid Irregular Settings. A corollary of our main theorem is that in a second price auction with a single anonymous reserve, namely a reserve drawn randomly from the distribution of $F_{\max }$ or a deterministic reserve of the monopoly price of $F_{\max }$, will achieve revenue that is a constant approximation to the geometric expectation of the maximum value. When the maximum value distribution is concentrated enough to have the geometric expectation is close to expectation it immediately follows that an anonymous reserve mechanism's revenue is close to that of the expected social surplus and hence the expected optimal revenue. 
Corollary 5. The second price auction with a single anonymous reserve achieves a revenue of at least $\frac{1}{e} \mathbb{G}\left[V_{\max }\right]$ for arbitrarily correlated bidder valuations.

Approximation via replicating buyers in Irregular Settings. When the auctioneer is unable to estimate the distribution of $V_{\max }$, and therefore unable to compute the reserve price, a well known alternative [1] to achieve good revenue is to recruit additional bidders to participate in the auction to increase competition. In our setting, recruiting a set of $n$ bidders distributed identically as the initial set of $n$ bidders (i.e. following joint distribution $F$ ) will simulate having a reserve drawn randomly from $F_{\max }$. In fact it performs even better than having a reserve one among the additionally recruited agents could be the winner and he pays the auctioneer, as against the reserve price setting. More formally, observe that in the setting with $2 n$ bidders, half of the revenue is achieved from the original $n$ bidders, and half from the new bidders (by symmetry). But the revenue from each of these parts is exactly that of the second price auction with a random reserve drawn from the distribution of $V_{\max }$. Hence, the revenue of this extended second price mechanism will be twice the revenue of a second price mechanism with a single random reserve drawn from the distribution of $V_{\max }$. This fact, coupled with our main theorem gives us the following corollary.

Corollary 6. The revenue of a second price auction with an additional set of bidders drawn from joint distribution $F$ is at least $\frac{2}{e} \mathbb{G}\left[V_{\max }\right]$.

Approximately Optimal and Efficient Mechanisms. Finally, we note that when the geometric expectation of $V_{\max }$ is close to its expectation, all our mechanisms (both the single pricing scheme, and Vickrey with a single reserve) are also approximately efficient.

Corollary 7. If $\mathbb{G}\left[V_{\max }\right]=c \mathbb{E}\left[V_{\max }\right]$, a single price drawn randomly from the distribution of $F_{\max }$ is simultaneously $\frac{c}{e}$ approximately revenue-optimal and $\frac{c}{e}$ approximately efficient.

Proof. Since expected social welfare of a pricing scheme is at least its expected revenue, we have:

$$
\mathbb{E}[\text { Social Welfare }] \geq \mathbb{E}[\text { Revenue }] \geq \frac{1}{e} \mathbb{G}\left[V_{\max }\right] \geq \frac{c}{e} \mathbb{E}\left[V_{\max }\right]
$$

\section{References}

1. J. Bulow and P. Klemperer. Auctions vs negotiations. American Economic Review, 86(1):180-194, 1996.

2. C. Daskalakis and G. Pierrakos. Simple, optimal and efficient auctions. In Proceedings of the 7th international conference on Internet and Network Economics, pages 109-121. Springer-Verlag, 2011. 
3. P. Dhangwatnotai, T. Roughgarden, and Q. Yan. Revenue maximization with a single sample. In Proceedings of the 11th ACM conference on Electronic commerce, EC '10, pages 129-138, 2010.

4. J. D. Hartline and T. Roughgarden. Simple versus optimal mechanisms. In $A C M$ Conference on Electronic Commerce, pages 225-234, 2009.

5. M. Paolella. Fundamental probability: A computational approach. John Wiley \& Sons, 2006.

6. O. Tamuz. A lower bound on seller revenue in single buyer monopoly auctions. Arxiv preprint arXiv:1204.5551, 2012. 